\title{
CASO 7-2012: ACIDOSIS LÁCTICA TIPO B1 SECUNDARIA A LINFOMA DE BURKITT.
}
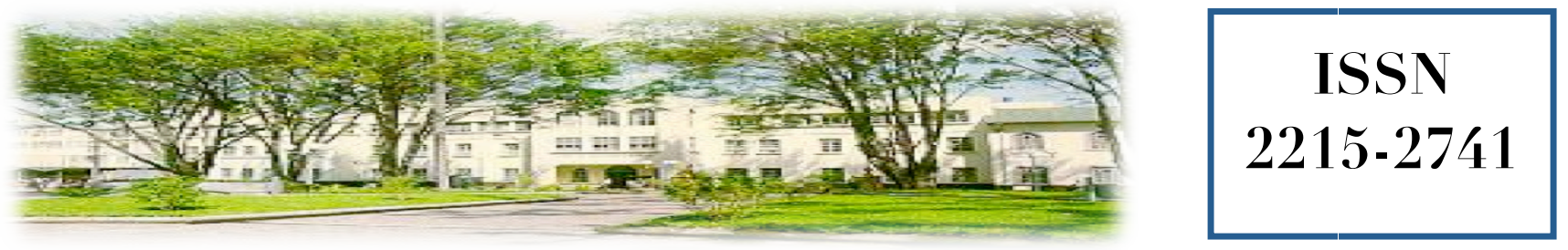

Hospital San quan de Dias. San José. Costa Riva. Fundado en 1845

\section{Reporte de Caso}

$\begin{array}{ll}\text { Recibido: } & 07 / 04 / 2012 \\ \text { Aceptado: } & 16 / 05 / 2012\end{array}$

Natalia Jiménez Brenes
${ }^{1}$
Jason Rojas Vega
Ibrahim Barguil Meza $^{3}$

${ }^{1}$ Estudiante de sexto año de Medicina. Escuela de Medicina. Universidad de Costa Rica. Departamento Clínico Hospital San Juan de Dios

${ }^{2}$ Estudiante Quinto Año de Medicina. Universidad de Costa Rica. Departamento Clínico Hospital San Juan de Dios

${ }^{3}$ Especialista en Medicia Interna. Jefe del Servicio de Medicina 1 HSJD. Profesor de la Cátedra de Medicina Interna. Escuela de Medicina UCR. Profesor del posgrado en Medicina Interna UCRCENDEISSS.

\section{RESUMEN}

La acidosis láctica es una complicación poco frecuente de las neoplasias malignas, que fue descrita por primera vez en pacientes con leucemia aguda en 1963. Desde entonces, se ha observado más a menudo, en particular en neoplasias hematológicas y rara vez en los tumores sólidos. Presentamos el caso de un hombre de 53 años con lactacidosis tipo B1 y linfoma de Burkitt gástrico, quien fue admitido al hospital con síntomas constitucionales, pérdida de peso, diarrea y melena. Los gases arteriales revelaron una acidosis metabólica con brecha aniónica aumentada (pH 7,278; $\mathrm{HCO}_{3}{ }^{-}$9,1 mmol/1, brecha aniónica 20) y concentración elevada de lactato $(15,5$ $\mathrm{mmol} / \mathrm{l})$. Se descartó causas de lactacidosis tipo
A como insuficiencia circulatoria, sepsis y choque. También se descartó causas comunes de acidosis láctica tipo $\mathrm{B}$, como infecciones, diabetes mellitus, falla renal, insuficiencia hepática, déficit de tiamina, fármacos y toxinas.

\section{PALABRAS CLAVE}

Acidosis láctica. Linfoma de Burkitt. Efecto de Warburg

\section{ABSTRACT}

Lactic acidosis is a rare complication of malignant neoplasms first described in patients with 
acute leukemia in 1963. Since then, there has been seen more often, particularly in hematological malignancies and rarely in solid tumors. We report the case of a 53 years old male with lactacidosis B1 and gastric Burkitt lymphoma who was admitted to the hospital with constitutional symptoms, weight loss, diarrhea and melena. Arterial blood gases revealed a metabolic acidosis with increased anion gap $\left(\mathrm{pH}: 7,278 ; \mathrm{HCO}_{3}{ }^{-}\right.$: 9,1 mmol/1; AG: 20) and elevated lactate $(15,5$ $\mathrm{mmol} / \mathrm{l})$. Other causes of lactacidosis, as circulatory failure, sepsis and shock were ruled out. Common causes of type B lactic acidosis were also excluded, such as infections, diabetes mellitus, renal failure, liver failure, thiamine deficiency, drugs and toxins.

\section{KEY WORDS}

Lactic acidosis. Burkitt's lymphoma. Warburg's effect.

\section{CASO CLÍNICO}

Masculino de 53 años de edad quien consultó por un cuadro de síntomas constitucionales, diarrea de baja tasa, criodiaforesis y melena. Se documentó hemoglobina en $4,3 \mathrm{~g} / \mathrm{dl}$, por lo que se trasfundió con 3 unidades de glóbulos rojos empacados y se ingresó para la realización de estudios. Asociado a este cuadro, refiere pérdida de peso de aproximadamente $17 \mathrm{~kg}$ en los últimos 9 meses y dolor epigástrico. Es tabaquista de 28.5 paquetes/año suspendido hace 3 años, y etilista importante durante 25 años suspendido hace 12 años. Tiene antecedente de gonorrea (hace 8 y 5 años) y antecedentes familiares de diabetes mellitus tipo 2, cáncer de mama y cáncer de tiroides.

Al examen físico se documentó presión arterial de $110 / 70 \mathrm{mmHg}$, taquicárdico, taquipneico, con $\mathrm{SpO}_{2}$ de 94\%, pálido, diaforético. Faringe eritematosa con secreción purulenta. Campos pulmonares con disminución del murmullo vesicular en base derecha y sibilancias de bajo tono difusas bilaterales. Abdomen poco depresible, doloroso a la palpación en ambos flancos, distendido. Hernia umbilical y hernia epigástrica. El tacto rectal mostró heces con sangre. Resto del examen físico dentro de límites normales.
Se documentó electrolitos séricos normales, glicemia al azar en $76 \mathrm{mg} / \mathrm{dl}$, elevación discreta de AST (134 UI/1), el resto de las pruebas de función hepática fue normal. Hiperglobulinemia $(4.3 \mathrm{~g} / \mathrm{dl})$ y DHL elevada (1136 UI/l).

US de abdomen: Engrosamiento parietal del TGI principalmente del estómago, adenopatías pericólicas, hepatomegalia con lesiones sugestivas de nódulos metastásicos, derrame pleural derecho y colelitiasis. Gastroscopía: al menos 4 lesiones induradas en cámara gástrica que asemejan pliegues engrosados sugestivos de linfoma gástrico. Se toman biopsias de estas lesiones. TAC de abdomen: quiste simple en segmento VIII hepático sin evidencia de nódulos metastásicos. Cámara gástrica con paredes engrosadas. No adenopatías retroperitoneales. Líquido libre de aspecto ascítico. Derrame pleural derecho. TAC de Tórax: Conglomerados adenopáticos en mediastino y axila derecha, derrame pleural derecho, engrosamiento del esófago distal. Hemograma y frotis de sangre periférica: $\mathrm{Hb}$ 9,9 $\mathrm{g} / \mathrm{dl}$, Hto $32 \%$, VCM 83 fl, plaquetas $145000 / \mathrm{mm}^{3}$, leucocitos $3000 / \mathrm{mm}^{3}$ (43\% neutrófilos, $40 \%$ linfocitos, $2 \%$ eosinófilos, $0 \%$ basófilos, $3 \%$ células en banda), anisocitosis ++ , basofilia difusa + , punteado basófilo + , microcitosis + , macrocitosis + , células en canasta y granulocitos vacuolados. Biopsias de mucosa gástrica: hallazgos histopatológicos y de inmunofluorescencia compatibles con linfoma de Burkitt.

\section{Cuadro 1. Resultado de gases arteriales.}

\begin{tabular}{|ccccccc|}
\hline Día & $\mathbf{1}$ & $\mathbf{2}$ & $\mathbf{3}$ & $\mathbf{6}$ & $\mathbf{8}$ & $\mathbf{1 1}$ \\
\hline $\mathbf{p H}$ & 7.31 & 7.28 & 7.32 & 7.40 & 7.42 & 7.47 \\
\hline $\begin{array}{c}\mathbf{p C O} \\
\mathbf{m m H g}\end{array}$ & 19.8 & 19.0 & 25.2 & 30.8 & 29.5 & 25.4 \\
\hline $\begin{array}{c}\mathbf{p O} \\
\mathbf{m m H g}\end{array}$ & 86.1 & 88.9 & 88.6 & 89.1 & 90.7 & 71 \\
\hline $\mathbf{S O}_{\mathbf{2}} \%$ & 94.6 & 94.7 & 95.2 & 95.2 & 96.3 & 9 \\
\hline $\mathbf{F I O 2}_{\mathbf{0}} \mathbf{0}$ & 21 & 21 & 21 & 21 & 21 & 21 \\
\hline $\begin{array}{c}\mathbf{H C O}_{3}^{-} \\
\mathbf{m m o l}_{\mathbf{l}}\end{array}$ & 9.7 & 9.1 & 13 & 18 & 18.8 & 18.7 \\
\hline
\end{tabular}

Fuente: Laboratorio Clínico Hospital San Juan de Dios.

\section{DISCUSIÓN}

La acidosis láctica se caracteriza por un aumento de la brecha aniónica, un aumento en la concentración sérica de lactato $>5 \mathrm{mmol} / \mathrm{l}$, y un $\mathrm{pH}$ menor de $7,3^{(1)}$, como en el caso del paciente que se presenta. Es una complicación poco frecuente 
de las neoplasias malignas principalmente en tumores malignos hematológicos y parece ser un marcador de mal pronóstico, independientemente del tratamiento que se ofrece y puede ser invariablemente fatal. ${ }^{(1)}$

El evento fisiopatológico principal en el trastorno ácido-básico es la acumulación del ácido láctico, el cual se comporta como la forma reducida del ácido pirúvico. ${ }^{(2)}$

Tradicionalmente se ha clasificado de acuerdo al proceso fisiopatológico que la produce ${ }^{(1)}$. La acidosis láctica tipo A se presenta en condiciones de hipoxia tisular mientras que la acidosis láctica tipo B ocurre en estados de perfusión normales, y se asocia a enfermedades adquiridas (subtipo B1), drogas y toxinas (subtipo B2) o a errores congénitos del metabolismo (subtipo B3). ${ }^{(3)}$

En condiciones adecuadas de oxigenación y aporte de nutrientes, una célula normal obtiene sus requerimientos de energía a partir de la producción glicolítica de piruvato, seguido por la conversión de piruvato a acetil-CoA por la enzima piruvato deshidrogenasa, que utiliza a la tiamina como cofactor. El Acetil CoA entra entonces en el ciclo del ácido tricarbónico (ATC) que tiene lugar en las mitocondrias. Este proceso resulta en la producción de 36 moléculas de ATP a partir de una sola molécula de glucosa. En ausencia de oxígeno, el ATC en las mitocondrias no puede ocurrir y por lo tanto, la célula tiene que utilizar la ruta glicolítica para hacer energía. ${ }^{(1)}$

La ruta glicolítica sólo suministra cuatro moléculas de ATP y el piruvato acumulado se convierte en ácido láctico. El ácido láctico se recicla de nuevo a glucosa en el hígado por la gluconeogénesis proceso conocido como el ciclo de Cori, en la que dos moléculas de ácido láctico producen una molécula de glucosa. ${ }^{(1)}$

Sin embargo, como fue señalado por Otto Van Warburg en 1924, las células tumorales cambian su maquinaria metabólica hacia un estado glicolítico, incluso en presencia de concentraciones normales de oxígeno (efecto Warburg). ${ }^{(1)}$

En el estado glicolítico durante el ciclo de Cori se utilizan seis moléculas de ATP para producir una sola molécula de glucosa y por lo tanto la célula consume más energía para regenerar la glucosa que se convertirá en lactato, lo cual parecería un proceso muy ineficiente para la célula, en términos de producción de energía. La razón de estos cambios metabólicos en una célula tumoral se desconoce con exactitud, sin embargo se plantea que estos cambios permiten a las células cancerosas adquirir y metabolizar nutrientes de una manera que favorece la proliferación y una producción más eficiente de ATP. ${ }^{(1)}$

Parece entonces, que la producción excesiva de ácido láctico en los pacientes con cáncer se contrarresta por el ciclo de Cori en el hígado. Por lo tanto, la mayoría de los pacientes con cáncer no manifiesta acidosis láctica tipo $\mathrm{B}$ y sólo en unos pocos pacientes este equilibrio metabólico se rompe, dando lugar a este trastorno. ${ }^{(1)}$

Los pacientes con Lactacidosis tipo B típicamente se presentan con hiperventilación y en ocasiones con hipotensión asociadas a taquicardia, debilidad muscular, vómitos, diarrea y estupor. ${ }^{(2)}$ Nuestro paciente presentó la mayoría de éstos síntomas los cuales mejoraron al mejorar su $\mathrm{pH}$ arterial.

En cuanto al tratamiento de la Acidosis Láctica, la literatura indica que el usar buffers exógenos para la alcalinización como el bicarbonato de sodio ha mostrado normalizar el $\mathrm{pH}$ arterial y atenuar el efecto deletéreo de la lactacidosis, sin embargo ha mostrado de igual manera una potenciación en la producción de lactato en pacientes con acidosis láctica crónica inducida por neoplasias. ${ }^{(2)}$ Al paciente en estudio se le administró $180 \mathrm{mEq} /$ día de bicarbonato de sodio a partir del día 5, con lo cual aumentó el $\mathrm{pH}$ arterial como consecuencia del aumento de $\mathrm{HCO}_{3}{ }^{-}$, sin embargo como es reportado por la literatura también hubo un aumento del lactato sérico como se puede observar en el cuadro 2 .

\section{Cuadro 2. Determinación de lactato sérico.}

\begin{tabular}{|c|c|c|}
\hline Día & 5 & 17 \\
\hline $\begin{array}{c}\text { Lactato } \\
\mathrm{mmol} / \mathrm{l}\end{array}$ & 12.9 & 15.5 \\
\hline
\end{tabular}
San Juan de Dios.

Otras modalidades de tratamiento son hemofiltración o hemodiálisis con baño de bicarbonato para normalizar el $\mathrm{pH}$, pero el nivel de lactacidemia permanece inalterable hasta que la quimioterapia funcione. Otra terapia es la adminis- 
tración de insulina con glucosa, lo cual incrementa la conversión de piruvato a acetil coenzima A y por consiguiente facilita la oxidación de lactato a piruvato, pero la mejoría suele ser discreta. $^{(2)}$ En nuestro paciente no se utilizó estas técnicas, ya que se presentó cierta mejoría clínica con la administración del bicarbonato de sodio

En el presente caso, es necesario hacer notar que a pesar de que al momento del ingreso, el paciente tenía múltiples factores de riesgo para sufrir hipoperfusión tisular (anemia y respuesta inflamatoria entre otros), en su evolución, la lactacidemia se mantuvo y más bien se elevó, a pesar de corregir los elementos antes citados.

\section{CONCLUSIONES}

A pesar de ser poco frecuente, las células tumorales malignas principalmente de neoplasias hematológicas pueden cambiar su metabolismo hacia el estado glicolítico, incluso en presencia de concentraciones normales de oxígeno. No se conoce con exactitud la razón de éstos cambios metabólicos pero se cree que se llevan a cabo para que las células cancerosas puedan adquirir y metabolizar nutrientes de una manera que favorezca la proliferación y una producción más eficiente de ATP.

Ante todo paciente con síntomas generalizados y acidosis láctica en ausencia de signos de mala perfusión tisular, se debe descartar la existencia de una neoplasia, habitualmente de origen hematológico, para instaurar tratamiento específico de forma precoz, único realmente efectivo en esta entidad. $^{(4)}$

\section{BIBLIOGRAFÍA}

1. Ruiz J. Singh A. Hart, P. Type B Lactic Acidosis Secondary to Malignancy: Case Report, Review of Published Cases, Insights into Pathogenesis, and Prospects for Therapy. The Scientific World Journal 2011; 1316-1324.

2. Loja, e. a. (Acidosis láctica severa y leucemia aguda. Anales de la Facultad de Medicina, Universidad Mayor de San Marcos 2004;65:49-55.

3. Vernon C. LeTourneau J. Lactic Acidosis: Recognition, Kinetics, and Associated Prognosis. Critical Care Clinics 2010; 255-283.
4. Fonseca A. (2004). Síndrome general con acidosis láctica de evolución fatal. Rev Clin Esp 2004;12:657-658. 